Service social

\title{
Manuel de gérontologie sociale 2, par Jean Carette, Boucherville, Gaëtan Morin éditeur, 1992, 187 pages.
}

\section{Georgette Béliveau}

Volume 42, numéro 3, 1993

Perspectives théoriques

URI : https://id.erudit.org/iderudit/706638ar

DOI : https://doi.org/10.7202/706638ar

Aller au sommaire du numéro

Éditeur(s)

École de service social de l'Université Laval

ISSN

1708-1734 (numérique)

Découvrir la revue

Citer ce compte rendu

Béliveau, G. (1993). Compte rendu de [Manuel de gérontologie sociale 2, par Jean Carette, Boucherville, Gaëtan Morin éditeur, 1992, 187 pages.] Service social, 42(3), 157-161. https://doi.org/10.7202/706638ar d'utilisation que vous pouvez consulter en ligne.

https://apropos.erudit.org/fr/usagers/politique-dutilisation/ 


\section{MANUEL DE GÉRONTOLOGIE SOCIALE 2}

Jean Carette

Boucherville, Gaëtan Morin éditeur, 1992, 187 pages.

Jean Carette présente un deuxième manuel de gérontologie sociale. L'auteur explique dans son introduction le pourquoi de ce deuxième volume ainsi que le cheminement qu'il a suivi. Chacun des 12 chapitres est précédé d'un encart dans lequel $M$. Carette donne les objectifs qu'il poursuit. On trouve en marge des notes intéressantes, originales et qui font bien la synthèse des idées. Les chapitres se terminent par un résumé, des exercices et une courte bibliographie. Le livre est centré sur l'intervention auprès des personnes retraitées et des personnes âgées elles-mêmes, mais la plupart du temps l'auteur s'adresse aux jeunes retraités pauvres ou démunis.

Le premier chapitre, intitulé "Se retirer et vieillir», se résume en trois idées principales:

- Le vieillissement peut entraîner des difficultés de communication.

- Ce sont surtout les conditions sociales de mise à la retraite qui sont préjudiciables.

- C'est sur ce plan que l'intervenant social doit agir et faire agir pour favoriser l'autonomie.

Bien qu'il soit parfois assez pessimiste, I'auteur explique bien certains volets de la réalité de la majorité des personnes âgées. Il donne sa vision, assez colorée et souvent juste, de la situation. II rappelle, par exemple, que la mémoire historique de la personne âgée peut être remplacée par celle de l'ordinateur, mais qu'il ne faut pas confondre cette mémoire avec l'expérience que seule la personne âgée possède. Si l'on songe aux systèmes experts, peut-on encore dire que l'expérience accumulée par les retraités ne saurait être mise à profit par les ordinateurs? Jean Carette se demande avec humour «s'il y a une vie avant la mort . II rappelle aussi, avec justesse, que "l'autonomisation » ne doit pas se faire au prix du désengagement de l'État.

Le deuxième chapitre, "Les fondements théoriques de l'intervention sociale en gérontologie», présente quatre modèles de perception de la société auxquels l'auteur associe différents types d'intervention. L'idée est originale, mais elle $n^{\prime}$ est pas suffisamment développée pour être vraiment utile aux intervenants. La deuxième partie du chapitre est consacrée au processus de changement auquel on relie l'idée d'autonomie. M. Carette définit cette dernière non pas comme l' «automobilité » ou l'autonomie physique ou même mentale, mais comme $I^{\prime}$ ' autogestion de la vie en société " ou la capacité de s'approprier sa vie.

Un troisième chapitre est consacré aux types d'intervention sociale. La première partie précise les cibles de l'intervention sociale et explique les trois niveaux: "le fonctionnement, les rapports de pouvoir et changer 
la vie(illesse) ». L'auteur ajoute des exemples à ses explications théoriques, ce qui rend le texte vivant. À la page 45, M. Carette donne les objectifs de l'intervention sociale et les résume dans un schéma clair et bien fait. Le reste du chapitre est consacré à l'explication de ce schéma.

Le chapitre qui suit est consacré aux étapes de l'intervention sociale. Le modèle utilisé est assez classique, mais il est enrichi d'exemples liés à l'intervention auprès des personnes âgées. La première étape s'intitule le cadrage de la situation. Elle a deux volets: I'origine de la demande et la collecte de l'information. On mentionne que la demande peut venir soit $d^{\prime}$ 'un retraité, soit d'un groupe de retraités, soit d'un intervenant, soit d'un établissement. En deuxième lieu, on procède à l'évaluation de la situation. L'auteur insiste alors sur le volet intellectuel "comprendre avec sa tête " et sur le volet affectif « une évaluation de personne à personne ». La troisième étape regroupe l'élaboration du plan d'intervention et le choix d'une ou de méthodes d'intervention. Vient en dernier lieu l'évaluation de l'intervention.

Le chapitre qui traite du rôle et de la place de l'intervenant social est centré sur le concept de changement auquel l'auteur relie celui de développement et d'autonomie de la personne âgée. L'intervenant doit adopter une attitude positive mais réaliste, car la réalité $n^{\prime}$ est ni toute rose, ni toute noire. L'auteur insiste ensuite sur l'établissement de rapports égalitaires entre la personne âgée et l'intervenant, ce qu'il qualifie d'un échange des savoirs, et en fait une condition du changement. II poursuit en insistant sur la nécessité de déculpabiliser le ou la retraitée, sur l'équilibre à tenir entre le problème individuel et la répétition de ce même problème chez plusieurs autres individus et sur les limites de l'intervention.

Le chapitre six s'intitule "La stratégie de prévention ». On pourrait lui donner comme sous-titre «Comment prendre sa retraite sans prendre un coup de vieux». La première partie est consacrée au passage à la retraite et aux divers changements qui surviennent à ce moment-là : la fin de la fonction de travailleur salarié, le démantèlement du réseau des collègues, la diminution du pouvoir d'achat, le nouveau contrat à deux et les relations avec la famille. Les objectifs de prévention se divisent en deux groupes: informer et former. Dans le premier point, l'auteur vise à faire connaître les étapes de la prise de retraite et les effets durables qui $\mathrm{s}^{\prime}$ ensuivent. Dans le second volet, $\mathrm{M}$. Carette fait le bilan des ressources accumulées grâce aux épargnes en matière de santé, de revenus, de relations, d'instruction et d'expérience, ce qui permet à la personne $d^{\prime}$ aménager sa retraite en conformité avec ses projets. Ce chapitre, sans être original, est un bon tour d'horizon des mécanismes d'adaptation à la retraite.

Le chapitre qui suit reprend l'idée de formation et traite des stratégies de formation: de l'individu au collectif. L'auteur définit la «stratégie de formation » comme "la stratégie qui a pour objectif d'engager l'individu 
dans une démarche personnelle d'accroissement de son autonomie, en le considérant d'abord comme un acteur de son développement ». Le thème de l'autonomie revient donc sur le sujet. Après avoir sévèrement critiqué I'intervention programmée qui oublie l'individu, l'auteur montre les limites de l'intervention-expertise qui oublie la collectivité, puis développe la stratégie "du dedans au dehors ". II la résume dans un tableau à la page 103. Cette intervention « vise à faire passer l'individu d'un rapport social subi à un rapport social maîtrisé grâce au soutien du groupe», idée qui a déjà été développée à quelques reprises dans le livre.

Le chapitre huit traite de la stratégie de formation: «Pour une action collective ". L'auteur reprend de façon originale, avec un groupe de personnes âgées défavorisées, une stratégie de formation bien adaptée à la clientèle visée. La première étape consiste en l'expression individuelle des difficultés de vie au moyen de dessins affichés sur une corde à linge. Ensuite, suit une recherche en petits groupes des origines sociales de ces difficultés. En dernier lieu, le groupe fait un "choix collectif d'une revendication prioritaire et la passation $d^{\prime} u n$ contrat $d^{\prime}$ action entre tous les membres du groupe ". L'idée d'une intervention de revendication revient ici et elle semble la seule possibilité offerte aux participants.

L'auteur aborde ensuite la stratégie de formation sous I'angle de la mobilisation. II relate les étapes d'une expérience de mobilisation de retraités. Au début des années 80 le gouvernement Mulroney avait promis en campagne électorale d'indexer de nouveau la pension de vieillesse. Arrivé au pouvoir, le premier ministre ne semble pas vouloir tenir sa promesse. Plusieurs groupes d'aînés se mobilisent et réussissent à obtenir ce qu'ils désirent grâce à leur stratégie. L'auteur termine ce chapitre par dix règles à observer dans de tels cas. L'expérience est bien relatée et donne un bon exemple de la théorie mise en évidence dans l'ouvrage.

Le chapitre dix traite de la stratégie de coanimation. L'auteur explique $d^{\prime}$ abord les similitudes et les différences entre l'animation des personnes âgées et l'animation en général. II en vient à définir la stratégie $d^{\prime}$ animation comme un ensemble d'initiatives permettant aux retraités qui le désirent de donner une nouvelle dimension individuelle et collective à leur vie de retraite, à partir de la libération, de l'expression et de la réalisation de leur projets personnels, mis en rapport avec les projets d'autrui.

M. Carette pose comme condition de réussite de la stratégie d'animation la connaissance des personnes retraitées et de leur milieu. II fait ensuite la différence entre la relation d'animation et la relation d'aide et ajoute quelques mises en garde à l'animateur "professionnel », car la "professionnalisation de l'animation devient un danger qu'il faut prendre au sérieux ». Enfin, le chapitre se termine sur une note humoristique avec 
la Chanson d'Anima. Ce chapitre, qui ne manque pas d'originalité, est novateur sous certains aspects, mais il répète un peu trop ce qui a été dit ailleurs dans ce volume et dans celui qui le précède.

Le chapitre onze a pour sujet la stratégie d'organisation que l'auteur voit comme un mal nécessaire. Le local doit devenir un lieu de retrouvailles que les aînés s'approprient, mais il doit rester un lieu transparent et ouvert. Quant à lui, le secrétariat est la mémoire du groupe. II permet, entre autres, d'être fidèle aux objectifs poursuivis et d'éviter les "détournements d'objectifs ». Les structures doivent demeurer au service des membres du groupe. L'auteur met en garde contre le recours aux subventions gouvernementales qui peuvent nuire à l'autonomie de l'organisation, étant donné que les bailleurs de fonds ont habituellement des règles de financement et demandent des comptes à ceux qu'ils pourvoient. De plus, les organismes gouvernementaux sont imputables aux différents ministères qui, eux-mêmes, sont redevables aux électeurs. Le chapitre se termine par une discussion sur la nécessité et le rôle des personnes-ressources: intervenants sociaux, experts et permanents.

Enfin, le dernier chapitre, "Pour une intervention conscientisante avec les retraitées et les préretraitées ", est consacré aux femmes retraitées, à leur condition sociale, à leur situation particulière et aux moyens d'une « intervention conscientisante ». La première partie trace un portrait social des femmes, portrait qui ne montre pas une situation enviable. Elles sont "pauvres, isolées, rejetées", sujettes au "mépris social des reines du foyer" et celles qui travaillent condamnées aux "ghettos d'emplois féminins". Elles "subissent violence et oppression». Enfin, " c'est le deuxième sexe! ». L'auteur reprend ensuite les propos qu'il a tenus au sujet de l'intervention, de la mobilisation et de la démarche vers l'autonomie en les appliquant aux femmes âgées.

Somme toute, il y a plusieurs idées originales et de bonnes synthèses dans cet ouvrage, mais aussi des répétitions dans le livre lui-même, $d^{\prime} u n$ manuel de gérontologie de l'auteur à l'autre et de ses manuels avec les autres écrits sur le sujet. Même si $M$. Carette fait des efforts pour être positif dans ces remarques, il s'en dégage un certain climat pessimiste un peu agaçant qui trace, des personnes âgées, un portrait de victimes. Celles-ci se reconnaissent-elles toutes et toujours dans ces esquisses? C'est probablement le cas de certains groupes sociaux. Les idées de changement de la société et de ses institutions, de conscientisation, de mobilisation reviennent sans cesse et avec insistance, mais les moyens concrets de faire les changements dans le respect des droits de l'ensemble des citoyens ne sont pas toujours aussi clairs. Il est naturel pour un auteur d'utiliser son ouvrage pour défendre ses idées personnelles sur un sujet. On ne saurait s'étonner de retrouver des prises de position idéologiques subtilement glissées entre les lignes ou entre les chapitres. Dans ce casci, la subtilité fait parfois défaut et on peut même se demander si l'auteur 
ne veut pas surtout prôner ses idées sur le sujet, sans qu'on soit bien sûr que ce soit aussi l'avis de l'ensemble des personnes retraitées et âgées elles-mêmes. Il n'en demeure pas moins que le livre est original, qu'il ne laisse pas le lecteur indifférent et que la formule pédagogique adoptée est intéressante.

Georgette BÉLIVEAU

École de service social

Université Laval 